\title{
Efficient Medical Image Enhancement using CLAHE Enhancement and Wavelet Fusion
}

\author{
Brij Bhan Singh and Shailendra Patel \\ Gargi Institute science and Technology \\ Bhopal, India,
}

\begin{abstract}
Medical image processing is a challenging field of research since the captured images suffers from the noise and poor contrast. The efficiency of the medical image processing depends on the quality of the captured medical images. Major factors for the low contrast medical images are age of capturing equipments, poor illumination conditions and inexperience of medical staff. Thus, contrast enhancement methods are used for improving the contrast of medical images before being used. In this paper an combination of the contrast limited adaptive histogram equalization (CLAHE) method and the wavelet based Fusion techniques are used for designing the efficient medical image enhancement method. Method is capable of adapting the Fusion rules adaptively for best enhancement results. First CLAHE image enhancement is used for improving the contrast of the medical images. then in second stage 2D Discrete wavelet transformation based adaptive image fusion is used for fusing the original and CLAHE output images. For testing the performance SNR and entropy are calculated and used as parameters. It is found that based on adaptive Fusion the visual content of the medical images are efficiently improved under all kind of capturing environments.
\end{abstract}

\section{Keywords}

Medical Image enhancement, RGB color spaces, Contrast limited Adaptive Histogram Equalization, Wavelet Fusion, Entropy.

\section{INTRODUCTION}

The medical images are accrued from different types of the sophisticated imaging camera systems and optical technologies Viz. MRI, CT scan, Somography and X-rays. Due to these techniques captured medical images suffers from noise and poor contrast issues. Therefore, contrast enhancement techniques [14, 15, and 17] are widely used to improving the quality of the medical images and their processing in the low illumination conditions. Contrast enhancement is the most common method of enhancing the image quality [14]. These methods basically improve the perceived difference between the image intensities in close proximity. Efficiency of the diseases identification through medical images depends on the efficiency of contrast enhancement. Therefore this paper focuses the problem of medical image enhancement using improved contrast performance of existing methods using wavelet based fusion. Wavelet fusion is widely used tool for image quality improvements [1, 3 and 4]. But since there are many wavelet based methods therefore it is very difficult to choose the particular fusing rules for specific medical image environment. Thus this paper proposes to design an adaptive medical image fusion method which can work efficiently for all images.

Image enhancements are broadly of two types Viz. spatial domain methods and transformation domain techniques. A spatial domain methods directly works on the image brightness thus are simpler. While most commonly used transform domain methods uses DWT [4] and DCT [1] for image enhancement Contrast Limited Adaptive Histogram Equalization (CLAHE) is widely used maximal entropy based spatial domain enhancement method [14] method is the most popular spatial domain enhancement method.

This paper takes advantage of both spatial domain and transforms domain techniques for improving the quality of the medical images. Paper proposes to enhance the input medical image suing spatial domain CLAHE method in RGB cooler space and then gray converted enhanced image and the original images are fused using wavelet based fusion in transform DWT domain.

Reaming part of paper is organized as follows: Section II have discussed the various existing enhancement methods and reviewed there works In section III describes the spatial domain CLAHE enhancement techniques in RGB space is described. In section IV Wavelet based fusion implementation in DWT domain is described in detail. Section V details result of proposed method for MRI application are presented and performance is companied. Section VI gives conclusion and future work.

\section{LITERATURE REVIEW}

Various methods are proposed in literature for enhancing the images such as methods using the image histogram, local region based enhancement, and transform domain methods. in this section paper reviews some of the relevant methods of enhancing the true color images.

\subsection{Review of Contrast enhancement methods}

A most popular method is Contrast Limited Adaptive Histogram Equalization (CLAHE) given by K. Zuiderveld [14] and have proposed to improve image contrast for medical imaging applications to overcome the amplification of noise problem and to improve the contrast. Etaa D. Pisano et al [15] have used contrast limited adaptive histogram equalization (CLAHE) method to improve the image quality. In last two decades various researchers used CLAHE as method for pre processing the different images..Hitam et al. [17] have presented a mixture of the CHAHE in RGB and HSV color spaces for enhancing the underwater images. They have used image fusion technique to produce the enhance image by fusing images in RGB and HSV color spaces. 
Table 1 Comparison of existing methods

\begin{tabular}{|c|c|c|c|}
\hline Author/ reference & $\begin{array}{l}\text { Method } \\
\text { Used }\end{array}$ & $\begin{array}{c}\text { Kind of images } \\
\text { (application areas) }\end{array}$ & Used Color spaces \\
\hline Peng.[3] & NSPDFB DWT & Medical images multimodle & $\begin{array}{l}\text { DWT based wavelet fusion method in } \\
\text { frequency domain }\end{array}$ \\
\hline Prateek et al [2] & DWT domain & $\begin{array}{l}\text { True colour } \\
\text { Images }\end{array}$ & $\begin{array}{l}\text { Scaling the DWT coefficient in } \mathrm{Y}-\mathrm{Cb}-\mathrm{Cr} \\
\text { Space }\end{array}$ \\
\hline Hatim et al. [5] & Spatial Domain & $\begin{array}{c}\text { Low contrast } \\
\text { Underwater images }\end{array}$ & $\begin{array}{c}\text { Using Fusion of CLAHE in RGB and } \\
\text { HSV colour space }\end{array}$ \\
\hline Our proposed work & $\begin{array}{c}\text { Spatial + DWT } \\
\text { Domain }\end{array}$ & $\begin{array}{c}\text { Different Medical images with } \\
\text { low contrast }\end{array}$ & $\begin{array}{c}\text { CLAHE + Pixel based adaptive wavelet } \\
\text { fusion rules }\end{array}$ \\
\hline
\end{tabular}

Vasile et al. [18] have proposed a hisogram equalization of color image enhancement using the adaptive neighborhood approach method was inconsistent. Irene et al [19] have used CIE-Lab color space for the detection of the optical disc in retinal images. In this paper SNR and mean square error are used as parameter to compare three enhancement techniques. The comparison of the reviewed methods of color image enhancements is presented in the Table 1. It is clear that the proposed method of image enhancement replaces $\mathrm{Y}-\mathrm{Cb}-\mathrm{Cr}$ with RGB color space and uses CLAHE and wavelet fusion.

\section{CONTRAST LIMITED ADAPTIVE HISTOGRAM EQUALIZATION (CLAHE)}

Another popular spatial domain method of contrast enhancement is CLAHE method [14, 17]. Method produces the optimal equalization in terms of maximum entropy and also limits the contrast of an image. The CLAHE method is very useful where the brightness requirement is high like as in geographical channels or underwater environments.

In this paper CLAHE method [14] is implemented for true color images. Equalization is implemented individually for all three RGB color spaces. These equalized RGB components are merged together to result the color equalized image [17]. CLAHE was originally developed for successfully enhancing the low contrast medical images [14]. Method partitions the images into related regions and finds the equalization to each region. This flattens the distribution of grey levels and thus makes hidden features of the image more visible. In this paper images are equalized in RGB color spaces individually. The basic algorithm for enhancing individual image regions by using the CLAHE technique is described below:

Step 1: Divide the each input image into a number of nonoverlapping contextual regions of equal size of the $8 \times 8$ blocks, each of which corresponds to the neighborhood of 64 pixels.

Step 2: Calculate the intensity histogram of each contextual regions.

Step 3: Set the clip limits for clipping the histograms, (for example $\mathrm{c}=0.002$ ). The clip limit is a threshold parameter for effectively altering the contrast of the image. Higher clip limits increases the contrast of local image regions thus it must be set to minimum optimal value.

Step 4: Modified the each histogram by the selecting a transformation functions.
Step 5: Each histogram is transformed in such a way that its height did not exceed the selected clip limit.

The mathematical expression for transformed gray levels for standard CLAHE method with Uniform Distribution can be given as

$$
g=\left[g_{\max }-g_{\min }\right] * P(f)+g_{\min }
$$

Where $g_{\max }=$ Maximum pixel value

$g_{\min }=$ Minimum pixel value

$\mathrm{g}$ is the computed pixel value

$P(f)=\mathrm{CPD}$ (Cumulative probability distribution)

For exponential distribution gray level can be adapted as

$$
g=g_{\min }-\left(\frac{1}{\alpha}\right) * \ln [1-P(f)]
$$

Where $\alpha$ is the clip parameter? CLAHE method operates on small regions in the image, called "tiles", rather than the entire image. Each tile's contrast is enhanced, so that the histogram of the output region approximately matches the histogram specified by the Distribution type. The CDF of Rayleigh distribution is given as;

$$
y=P(f(x \backslash b))=\int_{0}^{x} \frac{x}{b^{2}} e^{\left(\frac{-x^{2}}{2 b^{2}}\right)}
$$

Step 6: The neighboring tiles were combined using bilinear interpolation and the image grayscale values were altered according to the modified histograms.

An example of the CLAHE enhanced image is shown in the Figure 3 for lg-image16 with non uniform brightness. It is clear that the CLAHE method improves the information in the enhanced image by improving the contrast.
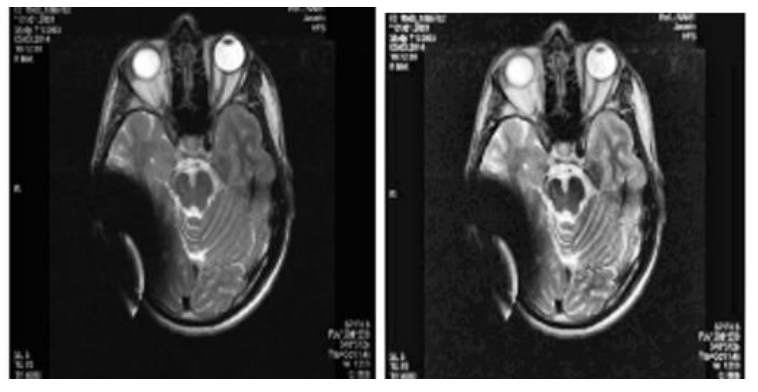

a) Input MRI Patient 1 image b) CLAHE enhanced image

Figure 2 Basic CLAHE enhancements 


\section{WAVELET BASED IMAGE FUSION TECHNIQUES}

Wavelet transform is first performed on source images. Then a fusion decision map is generated based on a set of fusion rules.

Then fused wavelet coefficient map can be constructed from the wavelet coefficients of the source images according to the fusion decision map. Finally the fused image is obtained by performing the inverse wavelet transform. The DWT based techniques became popular due to their multi-resolution properties. The main issue in wavelet fusion technique is the selection of decomposition level and the optimum wave filter

\subsection{Pixel Based Fusion Rules}

It is an intensity biased image fusion technique. In which we directly work on the intensities of individual pixels [23]. Let $\mathrm{A}(\mathrm{x}, \mathrm{y})$ and $\mathrm{B}(\mathrm{x}, \mathrm{y})$ are images to be fused, the decomposed low frequency sub images of $\mathrm{A}(\mathrm{x}, \mathrm{y})$ and $\mathrm{B}(\mathrm{x}, \mathrm{y})$ are $l \mathrm{~A}_{\mathrm{J}}(\mathrm{x}$, $\mathrm{y})$ and $l \mathrm{~B}_{\mathrm{J}}(\mathrm{x}, \mathrm{y})$. Decomposed high frequency sub images of $\mathrm{A}(\mathrm{x}, \mathrm{y})$ and $\mathrm{B}(\mathrm{x}, \mathrm{y})$ are $h \mathrm{~A}_{\mathrm{j}, \mathrm{k}}(\mathrm{x}, \mathrm{y})$ and $h \mathrm{~B}_{\mathrm{j}, \mathrm{k}}(\mathrm{x}, \mathrm{y})$. (j and $\mathrm{k}$ is the parameter of resolution, where $\mathrm{j}=1,2,3 \ldots . . J$ for every $j$, and $\mathrm{k}=1,2,3 \ldots k$ for every $k$ ) There are different pixel level fusions methods like as:

\subsection{Pixel Averaging (Method1)}

All the four sub bands of the fused image $\mathrm{F}$ is simply acquired by averaging the wavelet coefficients of source images A \& B.

$$
\mathrm{F}_{\mathrm{j}, \mathrm{k}}=\left(\mathrm{A}_{\mathrm{j} \mathrm{k}}+\mathrm{B}_{\mathrm{j}, \mathrm{k}}\right) / 2 \& \mathrm{~F}_{\mathrm{J}}=(l \mathrm{Aj}+l B j) / 2
$$

\subsection{Proposed adaptive Fusion techniques}

In this paper the wavelet based fusion is implemented on the original and the enhanced images for medical imaging applications. the min, max and averaging pixel level fusion rules are adopted based on the entropy analysis the comparison of the entropy of the faded images using all three methods are made and the best entropy is adopted as the adaptive fusion rules. The comparisons of wavelet decomposition for the original and enhanced CLAHE images are shown in the Figure 3. The input medical image and enhanced image is fused using the three pixel level fusion rules Viz. Pixel level Maxima, Pixel Level Minima and Pixel Level Averaging or mean.

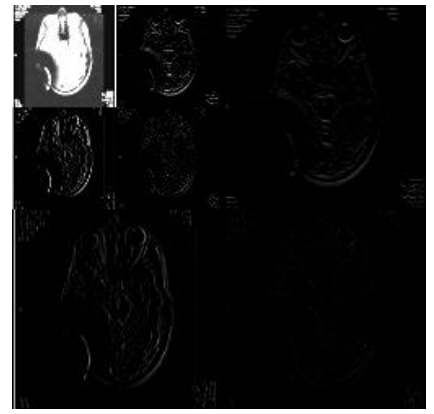

a) 2D wavelet decomposition of original; NRI image

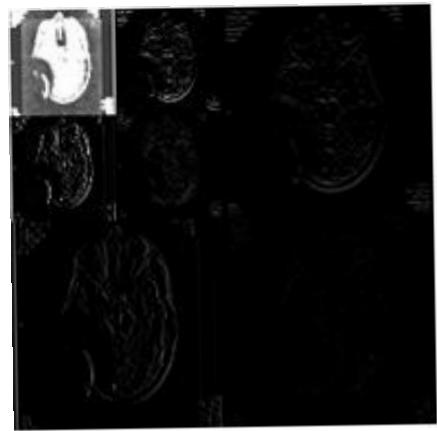

b) 2D wavelet decomposition of CLAHE Enhance image

Figure 3 DWT decomposition second level

\section{PROPOSED ADAPTIVE FUSION}

In this adaptive method, first input medical image is enhanced using the Contrast Limited Adaptive Histogram Equalization (CLAHE) method but implemented in RGB colour space separately. Both enhanced image and the input image are converted ot gray level image then wavelet decomposition of the input source images is performed up to level N. The Low pass and high pass sub-bands are then fused using different pixel level fusion methods is implemented for generating multiple enhanced images. Then the inverse wavelet transformation is performed to get full size fused images. The results of pixel level fused images are compared based on the entropy analysis. The results of maximum entropy are finally selected as the final enhanced image having maximum information. Proposed adaptive fusion method adopts the best pixel level fusion rules for enhancing the medical image quality based on entropy maximizations using the tri stage Entropy comparison as given here.

E1 $>$ E2 \& E1 $>$ E3 Image ${ }_{\text {out }}=$ Pixel level Maxima

$\mathrm{E} 1<\mathrm{E} 2 \& \mathrm{E} 2>\mathrm{E} 3$ then Image ${ }_{\text {out }}=$ Pixel level Minima

E3 $>$ E1 \& E3>E1 then Image ${ }_{\text {out }}=$ Pixel level Averaging

It is observed that adaptive fusion rule selection the efficiency of the medical image enhancement is improved since now the fusion results are environment independent

\section{RESULTS AND DISCUSSION}

Section presents the comparison of experimental results for the various mentioned color image enhancement methods. The input images used in this paper are shown in Figure 5.

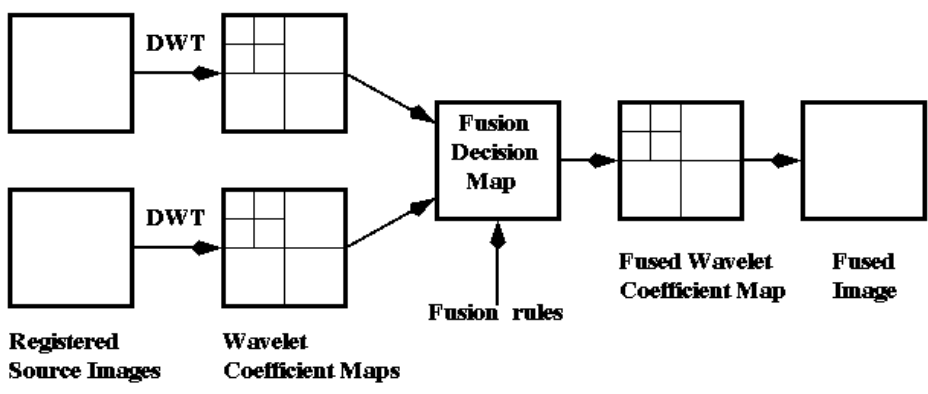

Figure 4 Wavelet based Image Fusion

Histogram equalization (HE) based method contrast limited adaptive histogram equalization CLAHE [5] improves the contrast and also preserves the brightness and entropy because method maximizes entropy. This method not only gives flat histogram but also enhances image contrast. 

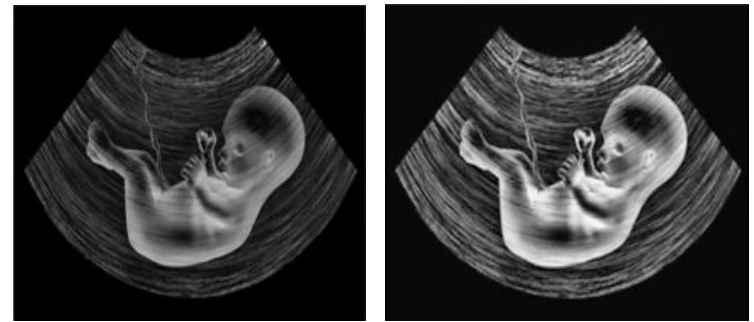

a) sonography image 1 b) CLAHE Enhanced image
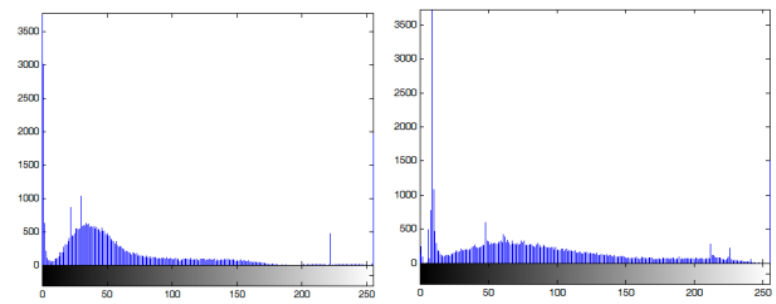

c) Histogram of original image d) CLAHE histogram

Figure 6 CLAHE Enhancement for Sonography_image_1
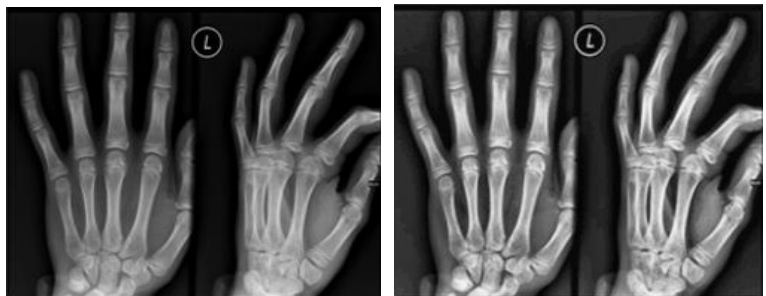

a) Original X_Ray imae 1 b) CLAHE Enhanced image
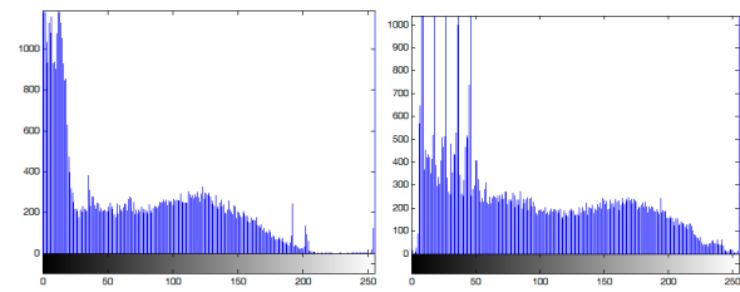

c) Histogram of original image d) Equalized histogram with CLAHE

Figure 7 CLAHE Enhancement for X_Ray_image

It is clear from the Figure 6 that enhanced Sonography image with CLAHE enhancement provides flat histogram distribution and describe the baby features more clearly. Therefore using CLAHE enhancement is used to generate the multi-focused image set from single input image as shown in Figure $6 a$ ) and $b$ ).

The fused results of various methods for Sonography image 1 is shown in the Figure 7.8. It can be observed that features in pixel level maxima fused image are more visible compared to others.

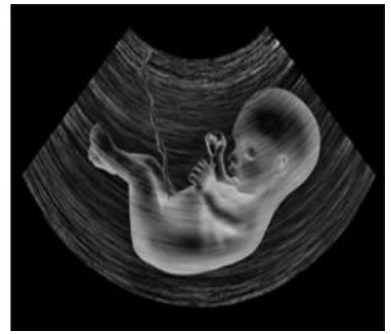

a) Original gray Sonography image 1

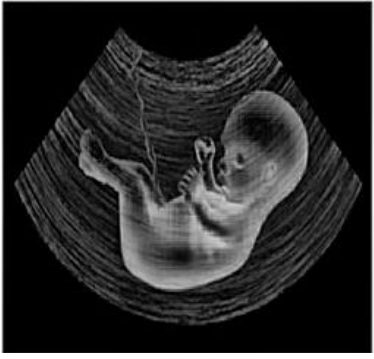

b) Minima Fused

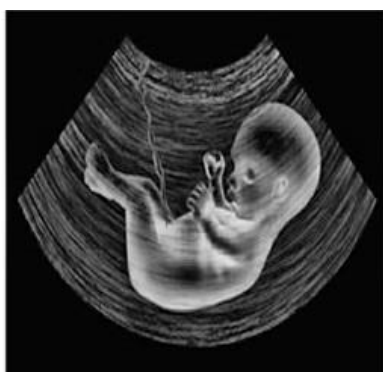

d) Average Fused e) Adaptive Fused image

Figure 8 Adaptive fusion results for Sonography image 1

Although variations in visual quality is not observed in few images by eyes thus the parametric evaluation based on the entropy analysis is performed as presented in Table 1 . Thus in dissertation evaluates and compare the performance of the various. Wavelet fusion rules for different medical images as shown in the Table 2.

Table 2 Comparison of the Entropy for Fusion methods

\begin{tabular}{|c|c|c|c|c|c|c|}
\hline S. No & Images & $\begin{array}{c}\text { Original } \\
\text { Image }\end{array}$ & Minima Fused & Maxima Fused & $\begin{array}{c}\text { Average } \\
\text { Fused }\end{array}$ & Proposed adaptive Fusion \\
\hline 1. & Sonogragy image 1 & 5.4592 & 5.4324 & 5.78036 & 5.7505 & 5.78036 \\
\hline 2. & Patient image 1 & 5.7536 & 6.3689 & 6.73445 & 6.6293 & 6.73445 \\
\hline 3. & Patient 2 CT Image & 5.3993 & 5.6074 & 5.7350 & 5.8761 & 5.8761 \\
\hline 4. & $\begin{array}{c}\text { Patient 2 MRI } \\
\text { image }\end{array}$ & 6.3862 & 6.3192 & 6.5969 & 6.6293 & 6.6293 \\
\hline 5. & Optical image 1 & 6.7966 & 6.2926 & 6.4643 & 6.3802 & 6.4643 \\
\hline 6. & X-Ray image & 7.0450 & 7.1402 & 7.4535 & 7.4824 & 7.4824 \\
\hline
\end{tabular}




\section{CONCLUSION}

Paper presents the comparison of image fusion methods for medical images. It is proposed to design the adaptive fusion rule based on entropy analysis.

The spatial domain methods CLAHE is used to generate the multi focused image set from single medical image. Performance of the three enhancement methods Viz. Pixel level Minima, Maxima and Averaging are compared based on Entropy analysis. It is found that our adaptive method works efficiently for all medical images and is capable of adapting the optimum fusion rule. The information content in the fused image is maximum since the entropy of the fused image is more than the input image in most of the images. Results are evaluated for six uniquely different medical imaging environments and it is found that the proposed adaptive fusion based method is independent of imaging environments.

\section{REFERENCES}

[1] Jayanta M., and Sanjit K. Mitra, "Enhancement of Color Images by Scaling the DCT Coefficients", IEEE Transactions on Image Processing, Vol. 17, No. 10, pp. 1783-1794, 2008

[2] Prateek S. Sengar, Tarun K. Rawat, Harish Parthasarathy, "Color Image Enhancement by Scaling the Discrete Wavelet Transform Coefficients", IEEE International Conference on Microelectronics, Communication and Renewable Energy (ICMiCR), 2013

[3] Peng Geng, Xing Su, Tan Xu, Jianshu Liu " Multi-modal Medical Image Fusion Based on the Multiwavelet and Non sub sampled Direction Filter Bank" International Journal of Signal Processing, Image Processing and Pattern Recognition Vol.8, No.11 pp.75-84 2015,

[4] Nayera Nahvi, Deep Mittal, "Medical Image Fusion Using Discrete Wavelet Transform", International Journal of Engineering Research and Applications Vol. 4, Issue 9( Version 5), pp.165-170 September 2014

[5] K. Zuiderveld, "Contrast Limited Adaptive Histogram Equalization", Graphics Gems IV, pp. 474-485

[6] S. Senthilkumar, S. Muttan, "Effective Multiresolute Computation to Remote Sensed Data Fusion" IEEE International Conference on Computational Intelligence and Multimedia Applications, 2007

[7] K. Kannan, S. Arumuga Perumal, K. Arulmozhi, "Area level fusion of Multi-focused Images using MultiStationary Wavelet Packet Transform “,International Journal of Computer Applications (0975 - 8887) Volume 2-No.1, May 2010

[8] Anjali Malviya, S. G. Bhirud, "Image Fusion of Digital Images", International Journal of Recent Trends in Engineering, Vol. 2, No. 3, November 2009

[9] Susmitha Vekkot, and Pancham Shukla, "A Novel Architecture for Wavelet based Image Fusion" Journal of World Academy of Science, Engineering and Technology, 57, pp. 32-3, 2009
[10] Jun Kong, Kaiyuan Zheng, Jingbo Zhang, Xue Feng, "Multi-focus Image Fusion Using Spatial Frequency and Genetic Algorithm",", IJCSNS International Journal of Computer Science and Network Security, Vol..8, No. 2, pp 220-224, February 2008

[11] J Gao, Z. Liu and T. Ren, "A new image fusion scheme based on wavelet transform Proc. ,3rd International Conference on Innovative computing, Information and Control, Dalian, China, Vol 2 ,June 2008

[12] Paresh rawat, Sapna Gangrade, Pankaj Vyas, "Implementation of Hybrid Image Fusion Technique Using Wavelet Based Fusion Rules", International Journal of Computer Technology and Electronics Engineering (IJCTEE) Volume 1, Issue 12011.

[11] Agung W. Setiawan, Tati R. Mengko, Oerip S. Santoso, Andriyan B. Suksmono, "Color retinal image enhancement using CLAHE", Proc. of 2013 International Conference on ICT for Smart Society (ICISS), pp. 1-3, 2013

[12] A.Saradha Devi, S. Suja Priyadharsini, S. Athinarayanan, "A Block based Scheme for Enhancing Low Luminated Images", International journal of Multimedia \& Its Applications (IJMA) Vol. 2, No. 3, pp. 49-61, 2010.

[13] Narasimhan K, Sudarshan C R, N. Raju, “A Comparison of Contrast Enhancement Techniques in Poor Illuminated Gray Level and Color Images", International Journal of Computer Application s0975 - 8887) Vol. 25, No .2, pp. 17-25, 2011 [14] K. Zuiderveld, "Contrast Limited Adaptive Histogram Equalization", Graphics Gems IV, pp. 474-485 1998.

[15] Etta D, Pisano, S. Zong, R. E Jhonston "Contrast limited adaptive histogram equalization image processing to improve the detection of simulated speculation in Dense Monograms", Journal of Digital Imaging, vol. 11, No. 4, pp 193-200, 1998

[16] Bhavana. Va , Krishnappa. H. Kb, "Multi-Modality Medical Image Fusion A survey" International Journal of Engineering Research \& Technology (IJERT) Vol. 4 Issue 02, February-2015

[17] Muhammad Suzuri Hitam,. Wan Nural Jawahir Hj Wan Yussof, Ezmahamrul Afreen Awalludin,, Zainuddin Bachok, "Mixture Contrast Limited Adaptive Histogram Equalization for Underwater Image Enhancement", IEEE international conf. 2013

[18] Vasile V. Buzuloin. Mihai Ciuc. Rangaraj M. Rangavvam. Loic Kjj. Coustantin Vertan, "Histogram equalization of colour images using the adaptive neighborhood approach", Proc. SPIE 3646, Nonlinear Image Processing X, 330, 1999.

[19] Irene Fondon, Mark J. J. P. van Grinsven, Clara I. Sanchez, Aurora Saez, "Perceptually Adapted Method for Optic Disc Detection on Retinal Fundus Images", Proc. of IEEE international conference pp. 279-284, CBMS 2011 